\title{
Initial experience with minimally invasive treatment of pilonidal sinus in children
}

\author{
Vojtech Dotlacil, Michal Rygl, Barbora Frybova \\ Department of Paediatric Surgery, Second Faculty of Medicine, Charles University and Motol University Hospital, Prague, Czech Republic
}

Videosurgery Miniinv 2021; 16 (2): 417-422

DOI: https://doi.org/10.5114/wiitm.2020.100714

\begin{abstract}
Introduction: Sinus pilonidalis $(S P)$ is an acquired inflammatory disease, which is relatively common in the paediatric population. Surgery is indicated in symptomatic patients. In 2017, minimally invasive pilonidal sinus treatment (EPSiT) was adapted to the paediatric population.

Aim: To evaluate the first experience with minimally invasive endoscopic treatment of SP (PEPSiT) in children and adolescents in the Czech Republic.

Material and methods: A retrospective review of all consecutive paediatric patients who underwent PEPSiT from November 2018 to February 2020. The monitored parameters were demographics, perioperative course of the disease, surgery, length of hospitalisation, postoperative complications, healing, disease recurrence, and follow-up.

Results: Seventeen patients were enrolled in the study. The median age at surgery was 17.1 years (range: 12.5-18). The subjects comprised $76 \%$ males, and the median body mass index was $25.6 \mathrm{~kg} / \mathrm{m}^{2}$ (range: $17-30.3 \mathrm{~kg} / \mathrm{m}^{2}$ ). Thirteen patients underwent previous surgical treatment (76\%) under local anaesthesia. The median duration of PEPSiT was $50 \mathrm{~min}$ (range: 30-85 min). The subjective evaluation of pain by patients on the VAS scale was 0 on the day of discharge. There were no postoperative complications up to the $30^{\text {th }}$ postoperative day. Two disease recurrences were successfully managed by re-PEPSiT. By the end of follow-up, 14/15 patients had healed. Two patients are still within 3 months of surgery, which is too soon to definitively evaluate possible recurrence of the disease.

Conclusions: These preliminary results show that PEPSiT is a highly promising method. It is safe and well-tolerated by patients (short hospital stay, quick return to normal life, low pain and analgesic consumption). Two recurrences of disease were treated by re-PEPSiT.
\end{abstract}

Key words: children, surgery, pilonidal sinus, minimally invasive technique, pediatric endoscopic pilonidal sinus treatment.

\section{Introduction}

Sacrococcygeal pilonidal sinus (SP) or pilonidal disease is a relatively common acquired inflammatory process affecting the intergluteal space above the coccyx. It was first described in 1833 by O. H. Mayo. The incidence of SP is $26 / 100,000$ individuals [1]. The risk factors are mainly associated with male gender, increased hair in the intergluteal region, being overweight, having a sedentary lifestyle, and local irritation [2]. The most common manifestations of the symptomatic pilonidal sinus (SPS) are pain, pressure in the sacrococcygeal region, and pathological secretion. In some individuals, complications may occur in the form of an abscess or recurrent acute or chronic infections with the development of fistulas with secretion. Symptomatic patients are indicated for treatment. The treatment

\section{Address for correspondence}

Vojtech Dotlacil MD, Department of Paediatric Surgery, Second Faculty of Medicine, Charles University and Motol University Hospital,

Prague, Czech Republic, e-mail: vojtech.dotlacil@seznam.cz 
may be both non-surgical and surgical. Non-surgical methods include application of phenol, fibrin glue, or a strictly conservative procedure (increased hygiene of the sinus area and regular hair epilation in the intergluteal space as a source of difficulties) [3-5]. There are many surgical procedures for the eradication of SPS, but none have yet become the surgical standard $[6,7]$. The reasons are mainly postoperative complications (14-30\%) and a high rate of disease recurrence (15-40\%) [8, 9]. Classical surgical methods often require long hospitalisation, are painful, and have a significant negative impact on the patient's quality of life for a long time in the postoperative period [10]. In 2014, Meinero et al. presented a newly developed minimally invasive surgical technique for the surgical treatment of SPS in adult patients, called endoscopic pilonidal sinus treatment (EPSiT), with promising treatment outcomes and very low invasiveness of the procedure [11]. This method has been adapted for use in the paediatric population and presented in 2018 by Esposito et al. [12]. This new method is ideal in terms of simple feasibility, short hospitalisation time, and minimal patient burden and pain, with good long-term results.

The aim of our work is to present the initial experience with minimally invasive surgical treatment of the symptomatic pilonidal sinus in children and adolescent in the Czech Republic.

\section{Aim}

To evaluate the first experience with minimally invasive endoscopic treatment of SP (PEPSiT) in children and adolescents in the Czech Republic.

\section{Material and methods}

A consecutive group of paediatric patients who underwent PEPSiT from November 2018 to February 2020 at the author's department were retrospectively reviewed. All patients signed informed consent. The patients could always choose between the classic or the PEPSiT surgery. The only exclusion criterion was an immunocompromising disease.

The reviewed parameters were demographic data, preoperative and postoperative course of the disease, use of antibiotics (ATB), surgery, postoperative pain, mobilisation, length of hospitalisation, postoperative complications, healing, disease recurrence, and length of postoperative follow-up.

\section{Definitions}

Healing: Complete skin closure of the surgical wound, without signs of inflammation, secretion, and pain, both subjectively and during clinical examination by a physician.

Recurrence: Lasting or recurrent secretion more than 3 months after surgery with infection or abscess formation and local pain and/or discomfort.

\section{Surgical procedure}

The surgical technique included two parts: diagnostic and therapeutic. The patient under general anaesthesia is placed in the 'jack-knife' position with abducted lower limbs and the intergluteal space is shaved. The first part of the method is the optical verification of the whole range of the sinus with an endoscope through external openings (Photos 1 A, B). The second/therapeutic phase consists of the removal of SPS content (hair, detritus, granulation tissue) and subsequently from the disruption of the fibrous capsule of the sinus with its excochleation. Finally, the walls and external openings of the sinus are electrocoagulated (Photo 2). Either a Meinero fistuloscope (Karl Storz $\mathrm{GmbH}$ ) or a resectoscope (Richard Wolf $\mathrm{GmbH}$ ) with an optical channel and a working and irrigation channel can be used (Photo 3). The whole procedure is described in the work of Meinero et al. from 2014 [11].

\section{Postoperative care}

Fluids are allowed $2 \mathrm{~h}$ after surgery and solid food after $4 \mathrm{~h}$. Mobilisation is allowed $4 \mathrm{~h}$ after the procedure. The patient is discharged on the first postoperative day with the original compressive dressing. After 2 days an outpatient control takes place during which parents are instructed about local care of the patient's surgical wound: regular shaving of the intergluteal space, washing of the external opening 3-4 times/day for at least 2 weeks with water and soap, and lavage of the cavity after SPS with Prontosan (B. Braun Medical Ltd.) solution at the same intervals. The same process continues for the next 2 weeks, but only 1-2 times/day (Photo 4). According to Esposito et al., use of laser epilation in combination with PEPSiT in the perioperative period is recommended for better results [13].

\section{Results}

Eighteen patients underwent PEPSiT surgery. Seventeen patients were enrolled in the study, 

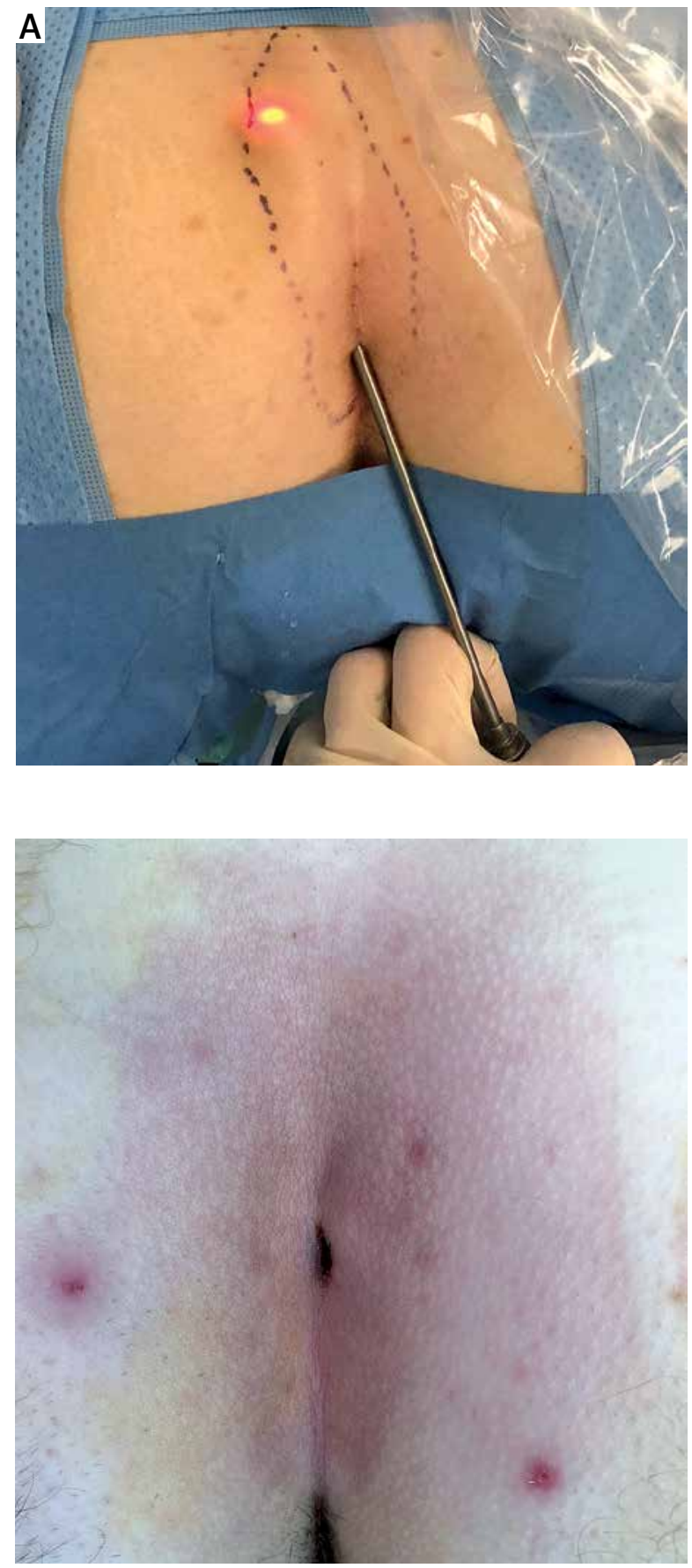

Photo 2. At the end of the PEPSiT

and 1 immunocompromised patient was excluded. The median age at the time of surgery was 17.1 years (range: 12.5-18). The subjects comprised $76 \%$ males, and the median body mass index was $25.6 \mathrm{~kg} / \mathrm{m}^{2}$ (range: $17-30.3 \mathrm{~kg} / \mathrm{m}^{2}$ ). Previous eradication attempts under general anaesthesia were performed in 3 (18\%) patients; this was always

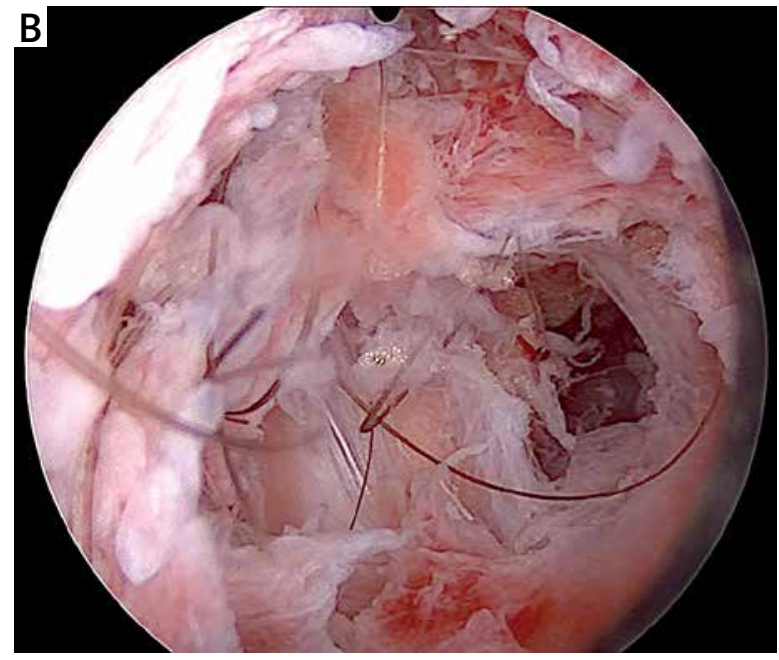

Photo 1. Endoscopic revision of SP: A - external view, B - interior view

excision with primary suture. Thirteen (76\%) patients underwent incision under local anaesthesia during the course of the disease followed by repeated local treatment. The median duration of disease before surgery was 10 months (range: 1-37). The median duration of PEPSiT was 50 min (range: 30-85). The median sinus length was $6 \mathrm{~cm}$ (range: 3-15), and the median number of fistulae from the sinus was $3(1-7)$. ATB prophylaxis was indicated in $15 \%$ of patients, and no postoperative antibiotic therapy was administered. The length of hospitalisation after surgery was 1 day in all patients. The pain intensity evaluated by the patient on a visual analogue scale (VAS) was 0 on the day of discharge. The median of pain on VAS scale was 0 (range: 0-3). Regular pain therapy by Paracetamol or Novalgin was ordered to first patients (35\%) in the postoperative period. Thereafter, it was only indicated if the pain intensity was equal to or greater than 3/10 on the VAS. There was no need for opioid analgesics in any patient. All patients were mobilised on the day of surgery. There was no postoperative complication according to the Dindo-Clavien classification by the $30^{\text {th }}$ postoperative day. By the end of the study, 14/15 patients had healed. 2/15 patients had recurrence of the disease and underwent re-PEPSiT - one is already healed. In 2 patients (2/17), follow-up was 


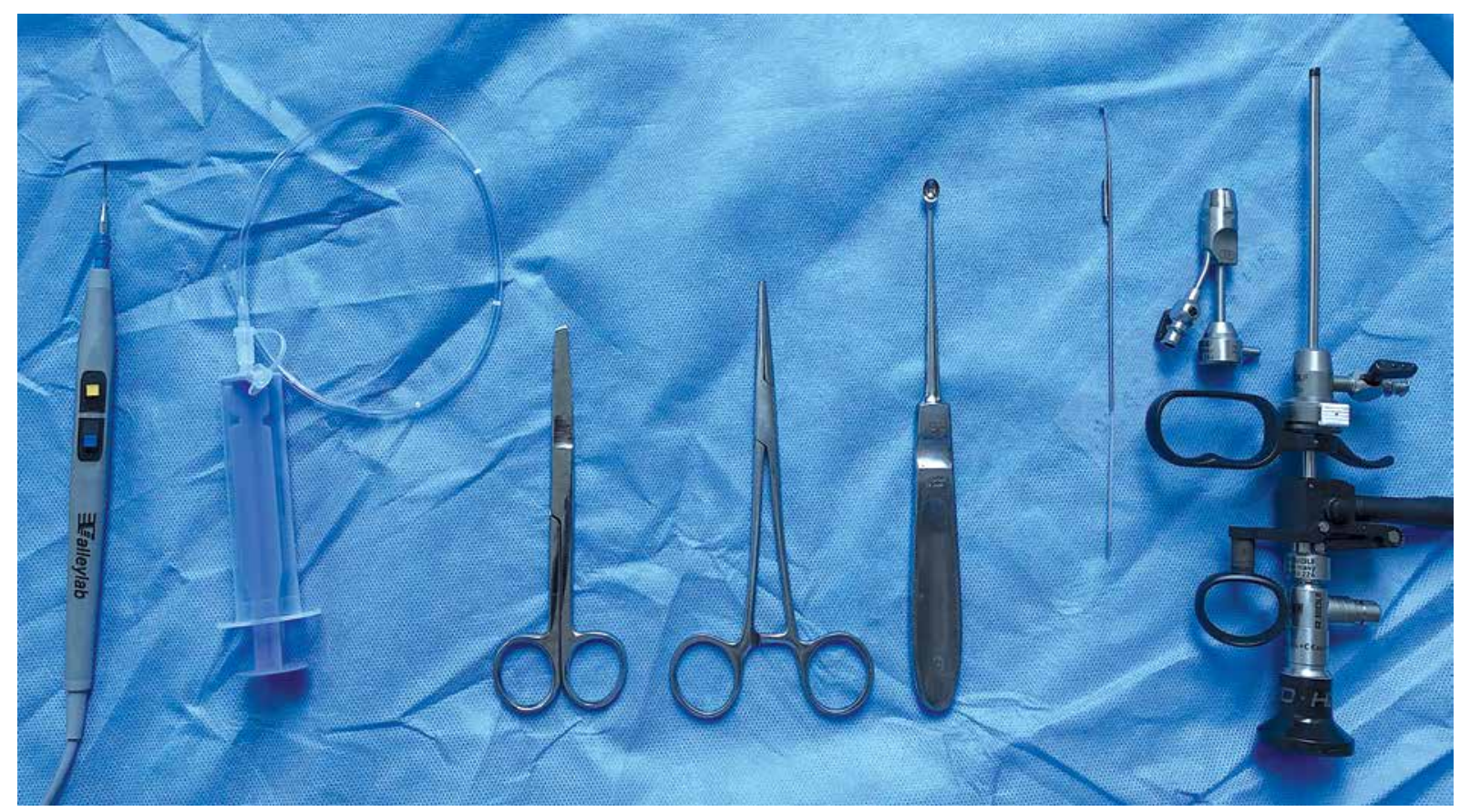

Photo 3. Instrumentarium

less than 3 months, making it impossible to assess possible recurrence. The median follow-up was 305 days (range: $54-475$ ).

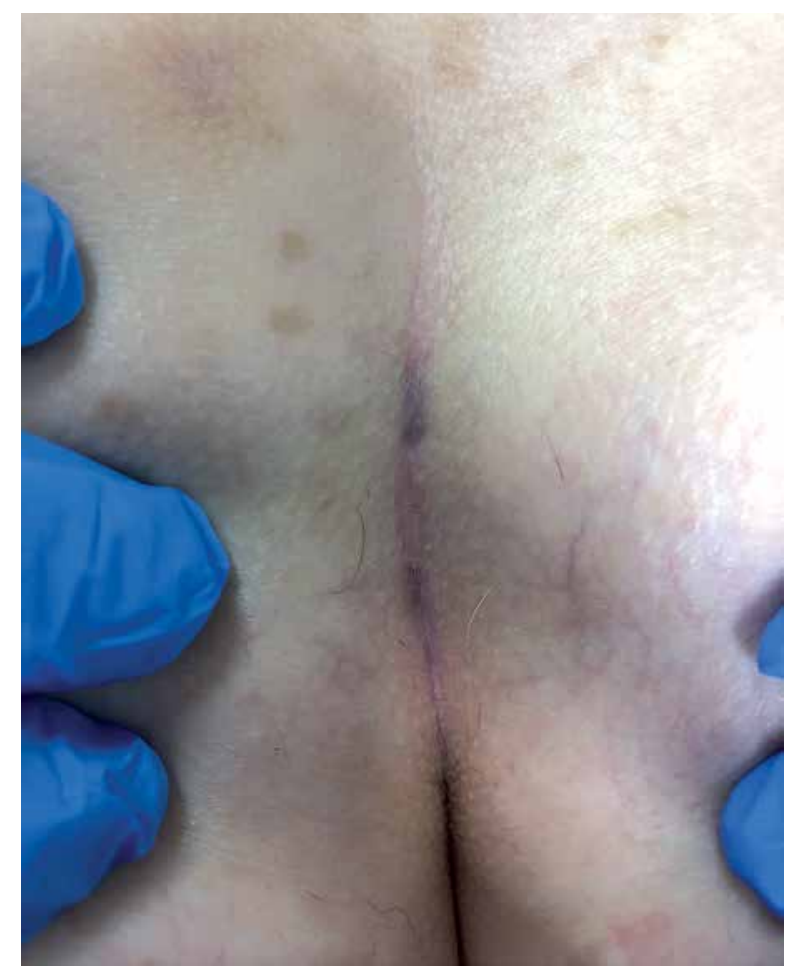

Photo 4. After healing

\section{Discussion}

This is the first presentation of minimally invasive surgical treatment of symptomatic pilonidal sinus, which has so far been performed in the Czech Republic only at the Department of Paediatric Surgery in Motol University Hospital. In our pilot study, we achieved $87 \%$ healing after first PEPSiT. Minimal invasiveness, short postoperative hospitalisation, and low requirement for analgesics were confirmed. In accordance with the literature data in our group, these are predominantly adolescent boys with excessive hair and a median BMI over $25 \mathrm{~kg} / \mathrm{m}^{2}$.

\section{Minimally invasive technique}

Recently, as in other branches of surgery, minimally invasive treatments have been introduced in the surgical treatment of SPS. The aim is to eradicate the pathological focus without the need for extensive excision and possible complications resulting from it. This can be done by laser eradication of SPS, which bears the designation SiLaC ${ }^{\circledR}$ (Sinus Laser ablation of the Cyst) [14] or the PEPSiT method, which is now used in the author's clinic.

This method can also be used successfully in recurrent SPS, as presented by Esposito et al. in 2019, where the patients were not significantly affected as in the case of classical re-excision, which must of- 
ten be more extensive and often afterwards requires a flap [15].

The PEPSiT method meets the requirements for the least invasiveness, with a low degree of pain, simple outpatient nursing, and the least possible exclusion of the patient from everyday life.

\section{Hospitalisation}

The length of postoperative hospitalisation after PEPSiT surgery was 1 day. According to a meta-analysis from randomised trials comparing SPS excision with primary suture versus Limberg flap surgery, the average length of hospitalisation was 5 days [16]. In 2011, Fike et al. published a retrospective study from their own department where a total of 120 patients were divided into two groups: the closed method (excision with primary suture or flap surgery) or the open method (marsupialisation, secondary healing), and the length of hospitalisation was reported (0.44 \pm 2.53 vs. $1.18 \pm 2.9$ days, $p=0.18$ ) [17]. Compared to the recently published PEPSiT data, the results in our study coincide with those published by Esposito et al. and the results published in a multicentre study by Prato et al. [18].

\section{Pain and need for analgesics}

According to standard surgical procedures at our department, regular pain therapy was ordered to first patients (35\%) in the postoperative period. According to the findings that these patients reported no pain and negated the need for analgesics, we evaluated the postoperative analgesic protocol, and only patients in whom the pain was equal to or higher than 3 were indicated for analgesics. At discharge, the pain was 0 in all patients according to the VAS scale. It follows from the above that the procedure is well-tolerated in terms of pain and with a minimal need for analgesics. Our pain management results are even lower than those reported in a study by Esposito et al. in which they compared pain after eradication with PEPSiT (mean: VAS 3.2) and the classical open technique (mean: VAS 6.8) [12].

\section{Complications and recurrence}

No intraoperative complications were observed in our group, and the number of postoperative complications up to the $30^{\text {th }}$ postoperative day according to Dindo-Clavien classification was $0 \%$. We observed two recurrences. The first recurrence occurred in the first patient, and we think that this recurrence was due to an atypical arrangement of SPS. The second recurrence was observed almost 4 months after the first surgery. A re-PEPSiT was performed in these patients; in the first patient the SPS is healed, in the second patient the healing is still in progress.

\section{Conclusions}

According to our preliminary results, minimally invasive PEPSiT treatment of symptomatic pilonidal sinus appears promising in children and adolescents. The surgical technique is relatively simple, easy to learn, easy to perform, and well-tolerated by the patient. However, for further evaluation, a larger, prospectively monitored set of patients for a longer period of time will be needed.

\section{Conflict of interest}

The authors declare no conflict of interest.

\section{References}

1. Yücesan S, Dindar H, Olcay I, et al. Prevalence of congenital abnormalities in Turkish school children. Eur J Epidemiol 1993; 9: 373-80.

2. Søndenaa K, Andersen E, Nesvik I, et al. Patient characteristics and symptoms in chronic pilonidal sinus disease. Int J Colorectal Dis 1995; 10: 39-42.

3. Chintapatla S, Safarani N, Kumar S, et al. Sacrococcygeal pilonidal sinus: historical review, pathological insight and surgical options. Tech Coloproctol 2003; 7: 3-8.

4. Hardy E, Herrod P, Sian T, et al. Fibrin glue obliteration is safe, effective and minimally invasive as first line treatment for pilonidal sinus disease in children. J Pediatr Surg 2019; 54: 1668-70.

5. Cevik M, Dorterler ME, Abbasoglu L. Is conservative treatment an effective option for pilonidal sinus disease in children? Int Wound J 2018; 15: 840-4.

6. Hardy EJO, Herrod PJ, Doleman B, et al. Surgical interventions for the treatment of sacrococcygeal pilonidal sinus disease in children: a systematic review and meta-analysis. J Pediatr Surg 2019; 54: 2222-33.

7. Orhalmi J, Sotona O, Dusek T, et al. Pilonidal sinus - possibilities surgical treatment. Rozhl Chir 2014; 93: 491-5.

8. Nasr A. A pediatric surgeon's 35-year experience with pilonidal disease in a Canadian children's hospital. Can J Surg 2011; 54: 39-42.

9. Enriquez-Navascues JM, Emparanza Jl, Alkorta M, et al. Metaanalysis of randomized controlled trials comparing different techniques with primary closure for chronic pilonidal sinus. Tech Coloproctol 2014; 18: 863-72.

10. Al-Khamis A, McCallum I, King PM, et al. Healing by primary versus secondary intention after surgical treatment for pilonidal sinus. Cochrane Database Syst Rev 2010; 2010: CD006213. 
11. Meinero P, Mori L, Gasloli G. Endoscopic pilonidal sinus treatment (E.P.Si.T.). Tech Coloproctol 2014; 18: 389-92.

12. Esposito C, Izzo S, Turrà F, et al. Pediatric endoscopic pilonidal sinus treatment, a revolutionary technique to adopt in children with pilonidal sinus fistulas: our preliminary experience. J Laparoendosc Adv Surg Tech A 2018; 28: 359-63.

13. Esposito C, Turrà F, Cerulo M, et al. Technical standardization of MIS management of children with pilonidal sinus disease using pediatric endoscopic pilonidal sinus treatment (PEPSiT) and laser epilation. J Pediatr Surg 2020; 55: 761-6.

14. Pappas AF, Christodoulou DK. A new minimally invasive treatment of pilonidal sinus disease with the use of a diode laser: a prospective large series of patients. Colorectal Dis 2018; 20: 0207-14.

15. Esposito C, Gargiulo F, Izzo S, et al. Pediatric endoscopic pilonidal sinus treatment: an effective procedure for children with recurrent pilonidal sinus disease after failed open surgery. J Laparoendosc Adv Surg Tech A 2019; 29: 981-6.

16. Horwood J, Hanratty D, Chandran P, et al. Primary closure or rhomboid excision and Limberg flap for the management of primary sacrococcygeal pilonidal disease? A meta-analysis of randomized controlled trials. Colorectal Dis 2012; 14: 143-51.

17. Fike FB, Mortellaro VE, Juang D, et al. Experience with pilonidal disease in children. J Surg Res 2011; 170: 165-8.

18. Pini Prato A, Mazzola C, Mattioli G, et al. Preliminary report on endoscopic pilonidal sinus treatment in children: results of a multicentric series. Pediatr Surg Int 2018; 34: 687-92.

Received: 13.07.2020, accepted: 12.09.2020. 